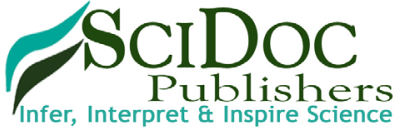

International Journal of Food Science, Nutrition and Dietetics (IJFS)

ISSN:2326-3350

\title{
Beneficial Effects of Moderate Intake of Red Wine on Glycemic Control, Lipid Profile and Pancreas Histoarchitecture in Diabetic Rats Fed on High Carbohydrate Diet
}

Research Article

Anyakudo $\mathrm{M}^{1 *}$, Olanubi $\mathrm{A}^{2}$

${ }^{1}$ Endocrinology/Metabolism and Clinical Nutrition Research Unit of Department of Physiology, Faculty of Basic Medical Sciences, University of Medical Sciences, Ondo City, Ondo State, Nigeria.

2 Department of Physiology, College of Health Sciences, Bowen University, Iwo, Osun State. Nigeria.

Abstract

Moderate alcohol consumption has been linked to improved cardiovascular and total mortality rates. However, the riskbenefit balance is controversial among moderate drinkers where evidence of type 2 diabetes was reported to be less prevalent. This experimentally-controlled designed study aimed to determine the effects of moderate red wine consumption on body anthropometry, glycemic and lipid profiles and pancreatic tissue histology in diabetic rats. Twenty eight male Wistar rats each weighing $\geq 200 \mathrm{~g}$ were randomly categorized into four experimental groups ( $\mathrm{n}=7$, each): Normal control fed with standard rat feed; Diabetic control (DC) fed with high carbohydrate diet; Diabetic on high carbohydrate diet treated with $4 \mathrm{ml} / \mathrm{kg}$ red wine (DR4) and Diabetic on high carbohydrate diet treated with $6 \mathrm{ml} / \mathrm{kg}$ red wine (DR6). Diabetes was inducted with freshly prepared alloxan monohydrate solution $(150 \mathrm{mg} / \mathrm{dL}$, intraperitoneally). Each rat was weighed twice weekly while the red wine was administered orally by cannula. Fasting blood glucose (FBG) concentrations were measured weekly in each rat in all groups and their means calculated. Lipid analysis was conducted at the beginning and the $5^{\text {th }}$ week of study period while the glycemic tolerance test using D-glucose ( $2 \mathrm{~g} / \mathrm{kg}$ wt) was conducted to construct the glycemic tolerance (GT) curves. Animals were sacrificed for pancreas extraction and histology. Graph Pad Prism and statistical program SPSS version 20 were used to analyze the data. $\mathrm{P}$ values $<0.05$ were considered significant. A significant $(\mathrm{p}<0.05)$ decrease in mean $\%$ weight gain was observed in red wine-treated diabetic groups: DR6 (9.14\%), DR4 (11.87\%) compared with DC (17.80\%). Wine-treated rats showed dose-dependent improved GT and significant decrease in mean \% FBG concentrations (DR4: $36.92 \%$ and DR6: $55.66 \%$ ) compared with the DC rats with elevated value (7.14\%) and impaired GT. Total cholesterol and triacylglycerides concentrations decreased significantly in wine-treated rats with elevated HDL levels and dose-dependent improved pancreas histoarchitecture. In conclusion, moderate consumption of red wine displayed dose-dependent beneficial organoprotective, hypoglycemic, anti-lipaemic and metabolic effects in diabetic rats.

Keywords: Diabetic Rats; High Carbohydrate Diet; Glycemic Control; Lipid Profile; Pancreas Histoarchitecture; Red Wine.

\section{Introduction}

Wine consumption and its use in health and medicine have long been established in history [1] until late $19^{\text {th }}$ and early $20^{\text {th }}$ century when medical research on alcohol began to cast doubt on the benefit of wine consumption as part of healthy living. However, in recent times studies have shown that moderate consumption of wine (preferably red wine) with meals confers positive health benefits provided the individual has no alcohol related health risk [2]. While attributing the potential health benefits of red wine to its constituent compounds including polyphenols, catechin and quercetin [4], studies have shown that red wine has a number of health benefits including cardioprotective, neuroprotective, immunoregulatory, antidiabetic and antiobesity functions [5]. The recommendation of red wine as part of diabetic diets has been fraught with controversy due to its constituent alcohol which has

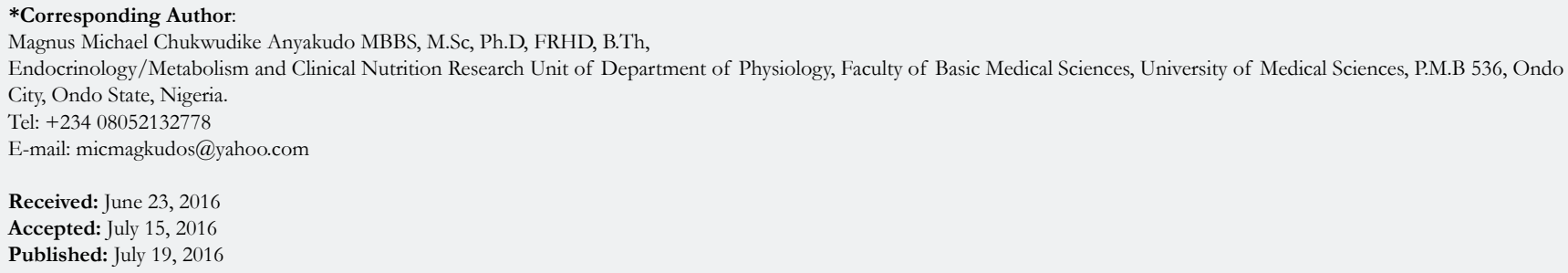

Citation: Anyakudo M, Olanubi A (2016) Beneficial Effects of Moderate Intake of Red Wine on Glycemic Control, Lipid Profile and Pancreas Histoarchitecture in Diabetic Rats Fed on High Carbohydrate Diet. Int J Food Sci Nutr Diet. 5(7), 313-318. doi: http://dx.doi.org/10.19070/2326-3350-1600056

Copyright: Anyakudo $\mathbf{M}^{\odot}$ 2016. This is an open-access article distributed under the terms of the Creative Commons Attribution License, which permits unrestricted use, distribution and reproduction in any medium, provided the original author and source are credited. 
been implicated in a number of health problems. However, some studies have suggested that moderate consumption of red wine may lower the risk of developing Type 2 diabetes mellitus problems [6] or delay the progression of the disorder and its complications [3]. The risk-benefit balance has been the focus of many researchers of interest when considering the recommendation of red wine in diabetic menu. Due to lack of long-term randomized studies, such balance has been fraught with controversy. Therefore, this experimentally controlled-designed study was carried out in diabetic rats to ascertain the suitability of moderate red wine recommendation in diabetic diets by determining its effects on glycemic and lipid profiles, body anthropometry and pancreas histoarchitecture.

\section{Materials and Methods}

\section{Experimental Animals and Design}

Twenty eight adult male Wistar rats (Rattus norvegicus) weighing $\geq$ $200 \mathrm{~g}$ were purchased from the disease-free stock of Olu farm, Ibadan, Oyo state, Nigeria. They were fed initially with standard rat chow and water ad libitum for the 2 weeks acclimatization in raised stainless steel cages with $6 \mathrm{~mm}^{2}$ mesh floor (to maintain same physical activity) and kept in a well ventilated animal house (at $23^{\circ} \mathrm{C}$ and a $12 \mathrm{~h}$ light and dark cycle). Replaceable numbered blotters papers were placed under each cage to catch the spilled diet that was measured to make up for the daily serving ration. After acclimatization, the experimental rats were randomly divided into four groups (Normal Control (NC) group; Diabetic group treated with $4 \mathrm{mls} / \mathrm{kg}$ red wine (DR4); Diabetic group treated with $6 \mathrm{mls} / \mathrm{kg}$ (DR6) red wine and Diabetic Control (DC) group) of 7 rats each such that each group had a close value of mean body weight (Table 1) and coefficient of variation. All animal weights were measured twice weekly and recorded. This study using experimental animals was conducted in accordance with the internationally accepted principles for laboratory animal use and care with the approval of the Animal Care and Use Review Committee of the Institution.

\section{Induction of Diabetes}

After 15 hours overnight fast following acclimatization, all rats except those in NC group were injected by single intraperitoneal injection of $150 \mathrm{mg} / \mathrm{kg}$ body weight of freshly prepared $2 \%$ alloxan monohydrate (Sigma chemicals, USA) dissolved in sterile $0.9 \%$ normal saline in a standard volumetric flask strapped with foil to prevent alloxan instability. Diabetes was confirmed 4-7 days later by use of glucometer (On Call Plus Blood Glucose Monitoring System, ACON Laboratories, Inc. San Diego, USA.) and compatible strips. Rats with Fasting Blood Glucose (FBG) level $>150 \mathrm{mg} / \mathrm{dl}$ were considered diabetic and used for this study since the level of serum glucose considered to be normal in rattus norvegicus ranges from $50-135 \mathrm{mg} / \mathrm{dL}$ [7]. Diabetes was allowed to stabilize for 5 days before exposure to experimental diets. Fasting blood glucose level of all rats in each experimental group was measured on weekly basis for the five-week study period.

\section{Animal feeding and Red Wine Administration}

The animals were fed according to the experimental design for the period of study with water ad-libitum. During acclimatization period, all animals were fed on standard rat chow and thereafter, following grouping, were exposed to high carbohydrate diet formulated based upon the standard diet formulas used to assess weight gain in rodents during commercial feeding studies. The red wine (Merlot varietal) containing 13\% alcohol purchased from a reputable store was administered to the rats in DR4 and DR6 groups via oropharyngeal cannula at $4 \mathrm{mls} / \mathrm{kg}$ and $6 \mathrm{mls} / \mathrm{kg}$ respectively.

\section{Oral Glucose Tolerance Test}

Animals in all groups were fasted overnight with free access to water before the day of experiment (last day of fifth-week) and were administered oral D-glucose load of $2 \mathrm{~g} \mathrm{~kg}^{-1}$ (dissolved in distilled water) by means of cannula after taking the initial fasting blood glucose (FBG) concentration. Thereafter, blood samples were withdrawn from the cordal vein of each animal (tail snipping) to determine the fasting blood sugar concentration at intervals of 30, 60, 90 and 120 minutes using glucose analyzer (On Call Plus Blood Glucose Monitoring System, ACON Laboratories, Inc. San Diego, USA.).

\section{Lipid Profile Analysis}

The lipid profile was conducted at the beginning and then 5 weeks later at the end of the study for comparison. Blood samples from the caudal veins were collected and transferred into the $\mathrm{k}_{3}$ EDTA (Ethylene Diamine Tetraacetic Acid) sample bottles. Samples were centrifuged at 3000 revolutions to obtain the plasma fractions which was kept in a refrigerator $\left(\right.$ at $-70^{\circ} \mathrm{C}$ ) until used and the sera obtained were used for the biochemical assay of the lipid profile. Plasma concentration of total cholesterol (TC), high density lipoprotein (HDL) and Triacylglycerol (TAG) were measured by the enzymatic colorimetric method after centrifugation using

Table 1. Effect of Red Wine on Body Weight (g) in Experimental Rats ( $n=7$ each/group).

\begin{tabular}{|c|c|c|c|c|}
\hline & \multicolumn{4}{|c|}{ Experimental Animal Categories } \\
\hline \multirow{2}{*}{$\begin{array}{c}\text { Parameters } \\
\text { Mean Body Weight (g) }\end{array}$} & Non-diabetic & \multicolumn{3}{|c|}{ Diabetic } \\
\cline { 2 - 5 } & NC (Normal Control) & $\begin{array}{c}\text { DR4 (4ml/kg } \\
\text { RW treated) }\end{array}$ & $\begin{array}{c}\text { DR6 } \\
\text { (6ml/kg RW treated) }\end{array}$ & $\begin{array}{c}\text { DC } \\
\text { (Diabetic Control) }\end{array}$ \\
\hline Initial & $210.40 \pm 11.33$ & $200.60 \pm 4.26$ & $205.04 \pm 4.19$ & $202.01 \pm 4.17$ \\
\hline Final & $242.10 \pm 13.44$ & $236.30 \pm 3.65$ & $229.30 \pm 3.59$ & $220.50 \pm 6.28$ \\
\hline Overall weight gain (\%) & 15.00 & $11.87^{*}$ & $9.14^{* a}$ & 17.80 \\
\hline
\end{tabular}

Values are expressed in mean \pm SEM. $*=$ significant $(\mathrm{p}<0.05)$ when compared with diabetic control. $a=$ not significant when compared with DR4. 
a dry-chemical automatic analyzer AU-5200 OLYMPUS (Randox Laboratories, San Francisco, USA). LDL level was determined by the Friedewald formula [8] as follows:

$\operatorname{VLDL}(\mathrm{mg} / \mathrm{dL})=\mathrm{TAG} / 5$

$\mathrm{LDL}(\mathrm{mg} / \mathrm{dL})=\mathrm{TC}-\mathrm{VLDL}-\mathrm{HDL}$

\section{Extraction and Histology of Pancreas}

At the end of the study, animals in all groups were anesthetized using Ethyl Ether in a glass dome and then dissected to extract the pancreas which was placed in 10\% formalin solution for a day. The pancreas of all the rats were then sectioned and histologically examined.

\section{Statistical Analysis}

The data obtained was computed, analyzed and summarized using Graph Pad Prisim and SPSS program version 20. Results (all mean values) are expressed as mean \pm SEM. Comparisons between groups were made using students't-test and one way analysis of variance (ANOVA). P-values $<0.05$ were considered statistically significant.

\section{Results}

\section{Effect of Red Wine on Body Weight}

The effect of red wine on body weight is presented in Table 1. A significant $(p<0.05)$ decrease in mean body weight gain ex- pressed in percentage was observed in red wine treated rats (DR4 - 11.87\%; DR6 - 9.14\%) compared with diabetic control (DC $-17.80 \%)$.

Effects of Red Wine on Blood Glucose Level and Glycemic Tolerance

The effects of red wine on the blood glucose concentrations and glycemic tolerance are shown in Table 2 and Figure 1 respectively below. Red wine caused significant $(\mathrm{p}<0.05)$ decrease in blood glucose concentrations at the end of study in a dose-dependent manner. On the glycemic tolerance curve, the red wine caused dose-dependent improved glycemic tolerance over that of the diabetic control as assessed by the incremental areas under the glycemic response curves.

\section{Effect of Red Wine on Lipid Profile}

The red wine effect on the lipid profile is shown in Table 3 below. Moderate intake of red wine caused dose-dependent improved lipid profile by significantly $(\mathrm{p}<0.05)$ reducing the triacylglycerides and low density lipoprotein concentrations with simultaneous increase in level of high density lipoprotein.

\section{Effect of Red wine on Pancreas Histoarchitecture}

Photomicrographs of the pancreas (Figures $2-5$ ) showed mild (DR6 rats) to moderate (DR4 rats) congestion of the islets cells interstitia compared with the diabetic control with severe interstitial congestion.

Table 2. Effect of Red Wine on Mean Blood Glucose Concentrations (mg/dL) in Experimental Rats (n=7 each/group).

\begin{tabular}{|c|c|c|c|c|}
\hline & \multicolumn{4}{|c|}{ Experimental Animal Categories } \\
\hline & \multicolumn{4}{|c|}{ Blood glucose concentration (mg/dL) } \\
\hline Duration (Weeks) & NC & DC & DR4 & DR6 \\
\hline 1 & $107.4 \pm 3.93$ & $162.4 \pm 1.70$ & $382.4 \pm 21.09$ & $293.2 \pm 27.91$ \\
\hline 5 & $72.8 \pm 0.92$ & $174.0 \pm 13.93$ & $241.2 \pm 35.85$ & $130.6 \pm 13.31$ \\
\hline Mean \% change & $32.20 \%$ & $7.10 \%$ & $36.90 \% *$ & $55.66 \% \mathrm{a}$ \\
\hline
\end{tabular}

Values are mean \pm SEM. $*$ and $\mathrm{a}=$ significant against diabetic control and diabetic $4 \mathrm{~mL} / \mathrm{kg}$ respectively.

Figure 1. Glycemic Tolerance Curves of Experimental rats ( $\mathrm{n}=7$ each/group).

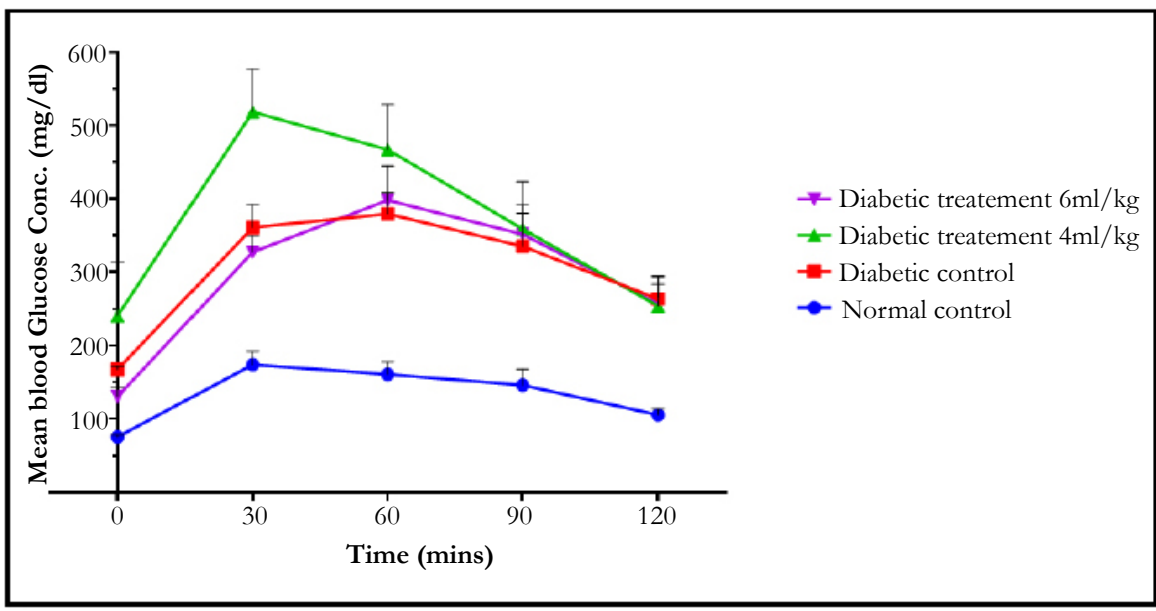


Table 3. Effect of Red Wine on Lipid Profile.

\begin{tabular}{|c|c|c|c|c|}
\hline \multirow{2}{*}{ Duration (weeks) } & \multicolumn{4}{|c|}{ Experimental groups } \\
\hline & DR4 & DC & DR6 & NC \\
\hline \multicolumn{5}{|c|}{ Total cholesterol (TC) mg/dl } \\
\hline 0 & $53.00 \pm 1.47$ & $52.20 \pm 3.05$ & $53.00 \pm 3.21$ & $52.23 \pm 2.20$ \\
\hline 5 & $50.50 \pm 1.50$ & $59.22 \pm 6.85$ & $48.05 \pm 0.95$ & $57.40 \pm 2.60$ \\
\hline \multicolumn{5}{|c|}{ Triacylglycerol (TG) mg/dl } \\
\hline 0 & $21.05 \pm 1.20$ & $20.45 \pm 1.00$ & $21.00 \pm 2.35$ & $20.05 \pm 2.30$ \\
\hline 5 & $20.24 \pm 1.00$ & $27.34 \pm 2.90$ & $19.44 \pm 1.00$ & $28.54 \pm 2.70$ \\
\hline \multicolumn{5}{|c|}{ High density lipoprotein cholesterol (HDL- C) mg/dl } \\
\hline 0 & $5.25 \pm 1.85$ & $5.15 \pm 1.05$ & $5.12 \pm 1.75$ & $5.20 \pm 1.75$ \\
\hline 5 & $15.35 \pm 2.50$ & $10.46 \pm 1.34$ & $18.00 \pm 2.85$ & $11.00 \pm 1.80$ \\
\hline \multicolumn{5}{|c|}{ Low density lipoprotein cholesterol (LDL- C) mg/dl } \\
\hline 0 & $43.54 \pm 1.25$ & $42.96 \pm 5.30$ & $43.68 \pm 2.25$ & $43.02 \pm 1.20$ \\
\hline 5 & $31.10 \pm 0.65$ & $43.29 \pm 1.20$ & $26.16 \pm 0.10$ & $40.69 \pm 0.03$ \\
\hline
\end{tabular}

Values are expressed in mean \pm SEM

Figure 2. Photomicrograph of the pancreatic tissue of a normal control rat showing normal histoarchitecture of the Islets cells (magnification x $400 \mathrm{H} \& \mathrm{E}$ Stain).

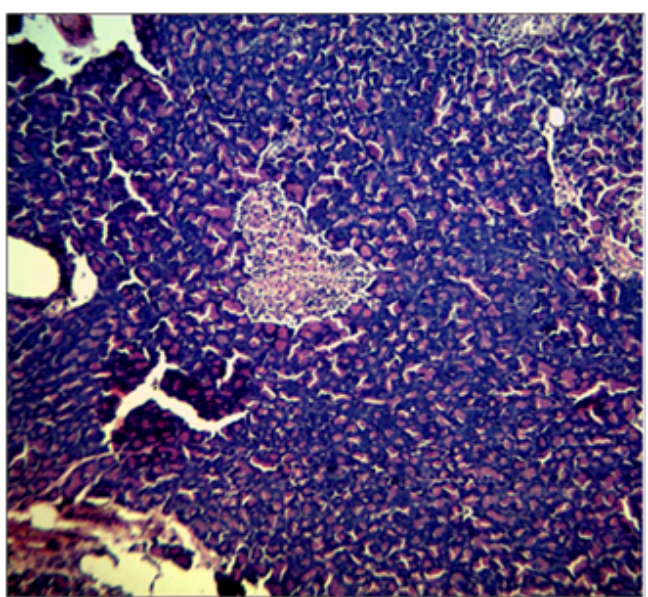

Figure 3. Photomicrograph of the pancreatic tissue of a diabetic rat (DR6) treated with wine (6ml/ $\mathrm{kg}) \mathrm{showing} \mathrm{mild} \mathrm{inter-}$ stitial congestion in the Islets cells (magnification $x 400 \mathrm{H} \& \mathrm{E}$ Stain)

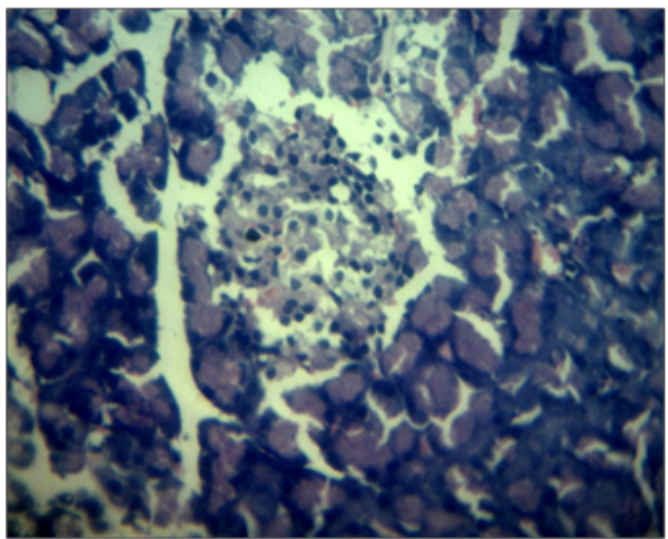


Figure 4. Photomicrograph of the pancreatic tissue of a diabetic control rat showing severe interstitial congestion in the Islets cells (magnification x $400 \mathrm{H} \& \mathrm{E}$ Stain)

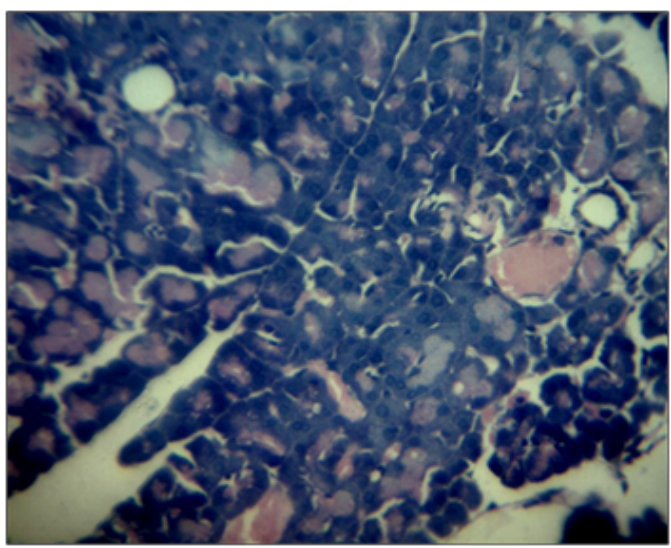

Figure 5. Photomicrograph of the pancreatic tissue of a diabetic rat (DR4) treated with wine $(4 \mathrm{ml} / \mathrm{kg}) \mathrm{showing} \mathrm{moderate}$ interstitial congestion in the Islets cells (magnification $x 400 \mathrm{H} \& \mathrm{E}$ Stain).

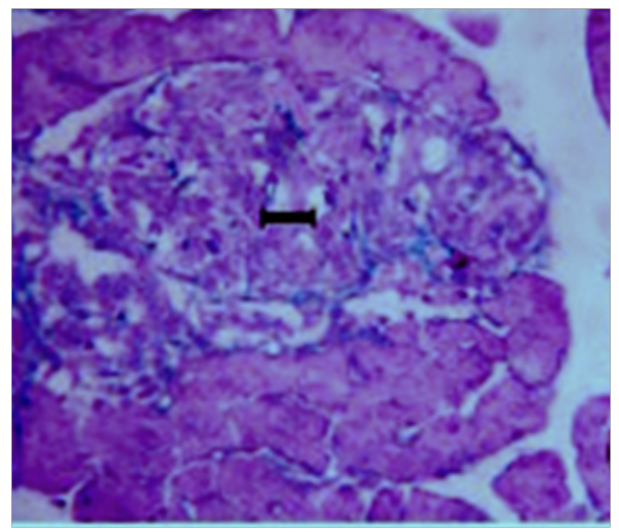

\section{Discussion}

This experimentally-controlled nutritional study determined and compared the effects of moderate consumption of measured portions of red wine on body anthropometry, glycemic control and tolerance, lipid profile and pancreas histoarchitecture in diabetic rats fed on high carbohydrate diet. The findings obtained from the study showed that red wine caused significant dose-dependent improved lipid profile, glycemic tolerance and control with significant reduction in weight gain and islets cells interstitial congestion.

The experimental rats in this study were fed with high carbohydrate diets to influence their weight gain such that the effect of the wine on body weight can be studied. The mean percentage weight gain after five weeks of study was significantly reduced in rats treated with wine compared with diabetic control. The dose-dependent effect of wine in this study was insignificant. This observed beneficial effect of red wine on weight gain when consumed moderately, reflects the possible antiobesity property of red wine as reported by other study using human subjects [5].

Alcoholic and non-alcoholic components of red wine have been shown to have significant effects on lipid variables $[10,11]$. The anti-lipaemic effect of red wine was observed in this study as evident by significant dose-dependent elevated HDL level and reduced levels of total cholesterol, LDL and triacylglycerol in DR4 and DR6 rats as shown in Table 3 compared with the diabetic control. Elevated triglycerides, LDL and cholesterol are potential cardiovascular risk factors for CHD and microvascular complications. Reduced HDL levels are both characteristics of diabetes mellitus [12] and a strong predictor of coronary heart disease [13]. Gepner et al., (2015) [2] in their study reported that moderate alcohol consumption improved cardiovascular and total mortality rate if a glass of red wine is consumed daily as part of a healthy diet while excessive alcohol consumption can result in higher blood pressure, increase cholesterol levels and weakened heart muscles.

Red wine has been reported recently to benefit people with type 2 diabetes (T2DM) due to observed reduced prevalence of T2DM among moderate drinkers [2]. This observation was also supported by the outcome of research study of Koppes et al., (2005) [6] who observed the decline in the risk of developing type $2 \mathrm{DM}$ among individuals consuming red wine with moderation. In this study, red wine displayed significant antihyperglycemic effect with improved glycemic profile on blood sugar concentrations in rats treated with red wine. These findings also agreed with the study of Montilla et al., (2005) [9] and Szkudelski and Szkudelska (2011) [20] which reported the hypoglycemic effect of moderately consumed red wine in human subjects. When translated into clinical practice, the benefits of drinking red wine should be weighed against potential risks in diabetic individuals. The lowering of blood sugar concentration by red wine as observed in this study 
may probably be mediated by reduction in glycemic and insulin responses as reflected in the glycemic tolerance curves in Figure 1 and reports of other studies [14, 15]. Another possible mechanism to explain this hypoglycemic effect of red wine may be achieved via delayed gastric emptying as reported in the study of Liljeberg and Bjorck (1998) [16]. Red wine in this study aids glycemic control in a short-term period. While the American Diabetes Association considered diabetic individual to decide appropriately the need to consume red wine in moderation, the American Heart Association however recommended individuals concerned to discuss their intention with their respective physicians. This study, which demonstrated the beneficial potential of red wine in diabetics if consumed with moderation, suggests the recommendation of red wine in diabetic diets if no contraindication exists.

Metabolic disorders such as Diabetes Mellitus and Obesity have been linked with disruption of the architecture of the pancreas [19]. The photomicrographs of the pancreas closely examined under high power magnification showed that red wine improved the pancreatic islet cells architecture by minimizing islets cells interstitial congestion. Scientific research has shown that polyphenols in general can stimulate $\beta$-cell growth and subsequently improve islet $\beta$-cell proliferation, survival and mass $[17,18]$. However, there is dearth of information on the effect of red wine polyphenols on the histoarchitecture of the pancreas in diabetics. This study therefore, provides useful information on the beneficial impact of moderate consumption of red wine on pancreatic tissue as revealed by the histological findings.

\section{Conclusion}

This study demonstrated the beneficial antiobesity, hypoglycemic and organoprotective effects of moderate intake of red wine on weight gain, lipid profile, glycemic control and pancreas histoarchitecture in diabetic rats fed on high carbohydrate diet. Moderate postprandial intake of red wine in dietary menu may be considered in diabetics without existing contraindication. However, the risk-benefit balance should be weighed prior to decision.

\section{Acknowledgement}

This work was carried out in collaboration between authors. Author MMCA designed, supervised, performed the analysis and wrote the manuscript of the study. Author OAF assisted in provision of essential materials, collection of samples data and analysis of results. Both authors read and approved the final manuscript.

\section{References}

[1]. Harding G (2005) A Wine Miscellany. Michael O'Mara Books Ltd, London, United Kingdom. 66-70.

[2]. Gepner Y, Golan R, Harman-Boehm I, Henkin Y, Schwarzfuchs D, et al., (2015) Effects of initiating moderate alcohol intake on cardiometabolic risk in adults with type 2 diabetes. Annals of Internal Medicine 163(8): 569-579. doi: $10.7326 /$ M14-1650.

[3]. Zahra B, Parvin M, Fereidoun A (2013) Dietary polyphenols as potential nutraceuticals in management of diabetes: A review. J Diabetes Metabol Dis 12(1): 43.

[4]. Robinson J (2006) The Oxford Companion to Wine. (3rd edn), Oxford University Press. Oxford. 569.

[5]. Lasebikan V (2011) Is red wine a good medicine? Nigerian Journal of Psychiatry 9(3): 10-16.

[6]. Koppes LJ, Dekker JM, Hendriks FJ, LM Bouter, Robert JH (2005) Moderate Alcohol Consumption Lowers the Risk of Type 2 Diabetes: A metaanalysis of prospective observational studies. Diabetes Care 28 (3): 719-25.

[7]. Harkness JE, Wagner JE (1993) Biology and clinical rabbits and rodents. (3rd edn), Sao Paulo, Roca. 48-55.

[8]. Friedewald WT, Levy RI, Fredrickson DS (1972) Estimation of the concentration of low-density lipoprotein cholesterol in plasma, without use of the preparative ultracentrifuge. Clin Chem 18(6): 499-502.

[9]. Montilla P, Barcos M, Muñoz MC, Bujalance I, Munoz-Castaneda JR, et al., (2005) Red Wine Prevents Brain Oxidative Stress and Nephropathy in Streptozotocin-induced Diabetic Rats. J Biochem Mol Biol 38(5): 539-544.

[10]. Zang M, Xu S, Maitland-Toolan A, Zuccollo A, Hou X, Jiang B (2006) Polyphenols stimulate AMP-activated protein kinase, lower lipids, and inhibit accelerated atherosclerosis in diabetic LDL receptor-deficient mice. Diabetes 55(8): 2180-2191.

[11]. Guilford J, Pezzuto J (2011) Wine and Health: A Review. Am J Enol Vitic 62 (4): 471-486.

[12]. Cowie CC, Howard BV, Harris MI (1994) Serum lipoproteins in African Americans and whites with non-insulin-dependent diabetes in the US population. Circulation 90(3): 1185-1193.

[13]. Gordon T, Castelli WP, Hjortland MC, Kannel WB, Dawber TR, et al., (1977) High density lipoprotein as a protective factor against coronary heart disease. Am J Med 62(5): 707-714.

[14]. Ebihara K, Nakajima A (1988) Effect of acetic acid and vinegar on blood glucose and insulin responses to orally administered sucrose and starch. Agric Biol Chem 52(5):1311-1312.

[15]. Leeman M, Ostman E, Bjorck I (2005) Vinegar dressing and cold storage of potatoes lowers postprandial glycaemic and insulinaemic responses in healthy subjects. Eur J Clin Nutr 59(11): 1266-1271.

[16]. Liljeberg H, Bjorck I (1998) Delayed gastric emptying rate may explain improved glycemia in healthy subjects to a starchy meal with added vinegar. Eur J Clin Nutr 52(5): 368-371.

[17]. Fu Z, Liu D (2009) Long-term exposure to genistein improves insulin secretory function of pancreatic beta-cells. Eur J Pharmacol 616(1): 321-327.

[18]. Fu Z, Zhang W, Zhen W, et al., (2010) Genistein induces pancreatic betacell proliferation through activation of multiple signaling pathways and prevents insulin-deficient diabetes in mice. Endocrinology 151(7): 3026-3037.

[19]. Vessby B (1994) Dieatary Carbohydrates in diabetes mellitus. J Am Clin Nutr 59(3): 7425-7465

[20]. Szkudelski T, Szkudelska K (2011) Anti-diabetic effects of resveratrol. Ann NY Acad Sci 1215: 34-39. 